\title{
STEM CELLS: AN APPROACH FOR TREATMENT OF ATHEROSCLEROSIS
}

\author{
VINOD KUMAR JATAV, SUNITA SHARMA* \\ Assistant Professor, Department of Biotechnology, Madhav Institute of Technology and Science, Gwalior - 474005, Madhya Pradesh, India. \\ Email: sunitasharma75@yahoo.co.in
}

Received: 21 September 2017, Revised and Accepted: 02 July 2018

ABSTRACT

The contribution of stem cells to control atherosclerosis progression and vascular remodeling after injury in humans is established; however, many details are still unknown. The success with stem cell therapies in the treatment of hematological disorders over the past four decades enhanced our understanding of the physiology of vascular remodeling and motivated towards regenerative medicine and therapeutic restoration of the damaged organ. Stem cells isolated from bone marrow are currently being tested for their therapeutic potential in a variety of clinical conditions including cardiovascular injury, kidney failure, cancer, neurological, and bone disorders. The main focus of this review paper is to reveal the role of different types of stem cells such as embryonic stem cell, adult stem cell, smooth muscle progenitor cells, endothelial progenitor cells, mesenchymal stem cells, and vascular smooth muscle cells and find out possibility to use them for the treatment of atherosclerosis as well as to reduce atheroma formation.

Keywords: Stem cell, Cardiac diseases, Atherosclerosis, Bone marrow, Transplantation.

(C) 2018 The Authors. Published by Innovare Academic Sciences Pvt Ltd. This is an open access article under the CC BY license (http://creativecommons. org/licenses/by/4. 0/) DOI: http://dx.doi.org/10.22159/ajpcr.2018.v11i10.22690

\section{INTRODUCTION}

Atherosclerosis leads to cerebrovascular disease and coronary artery disease through slow progression of lesion formation and luminal narrowing of arteries. Plaque rupturing and thrombosis are the most common symptoms of the cardiovascular disease which are quite apparent to acute coronary syndrome, myocardial infarction, or stroke. The other significant signs of atherosclerosis are characterized by a chronic inflammatory process of the arterial wall that occurs at predilection sites such as branch points with the disturbed laminar flow [1]

Atherosclerosis is characterized by slowly growing, silent lesions known as stable plaques or may be associated with the formation of unstable plaques [2]. A stable plaque is associated with smooth fibrous coronary artery plaques, whereas unstable plaques lead to acute myocardial infarction (AMI), and sudden cardiac death is almost invariably associated with irregular or ruptured plaques [3]. The pathogenetic role of macrophages at the site of cap rupture in patients with fatal AMI is very well understood. Similarly, patients with carotid artery disease, plaque irregularity and rupture are associated with cerebral ischemic events and patients with irregular or ulcerated plaques have a higher risk of ischemic stroke irrespective of the degree of stenosis of the vessel lumen [4].

\section{ATHEROSCLEROSIS}

Prevalence

Cardiac patients are increasing globally and the number of patients awaiting transplantation continuously increasing. The major limitation for organ transplantation is a shortage of available deceased organ donors [5]. About $30 \%$ of all global death was attributed to cardiovascular diseases in 2008. The death rates are also higher in low- and middle-income countries as over $80 \%$ of all global death caused by cardiovascular diseases occurred in these countries and by 2030 , millions of people will die from cardiovascular diseases each year. It is reported that $60 \%$ of the world's cardiovascular disease burden will occur in the South Asian subcontinent despite accounting for $20 \%$ of the world's population [6].

Responsible factors of atherosclerosis

A large number of factors can cause atherosclerosis such as genetic predisposition, oxidative stress, sex hormones, toxins, mutagens, dietary fat intake, and cigarette smoking, non-specific injury, infections and inflammation, DNA instability, cell proliferation and clonal expansion, apoptosis and telomerase [7].

The role of genetic factors for the inheritance of atherosclerosis has been understood, and genetic variation, such as mutations or common polymorphisms has been shown to be involved in modulation of a range of risk factors, such as plasma lipoprotein levels, inflammation, and vascular calcification [8]. In recent years atherosclerotic diseases have been identified associated with monogenetic diseases, candidate genes, genetic polymorphisms and susceptibility loci, and the number is going to increase very rapidly [9]. The atherosclerotic condition is caused due to a decreased level of apolipoprotein B-100, lipoprotein retention within the arterial wall, efflux of cholesterol and other harmful lipids from plaques, and emigration of lesional foam cells followed by entry of healthy phagocytes that remove necrotic debris and other plaque components. Plaque milieu is improved when cellular lipid efflux and foam cell emigration occur. Each process involve specific molecular mediators, many of which have been identified [10].

Macrophages play an important role in atheroma formation including lesion initiation, progression, and complication. In general, macrophages are initially recruited to the artery wall as monocytes to ingest oxidized lipoproteins through scavenger receptors, and some of the macrophages can die within the atheroma through oncosis and/or apoptosis, which results in the formation of paucicellular lipid core. They produce many proteases such as matrix metalloproteinases which degrade the extracellular matrix comprising fibrous cap [11].

\section{Disease progression factors in atherosclerosis}

Disease progressive factors of atherosclerosis are transforming growth factor-beta pathway, peptide growth factors, epidermal growth factor, receptor, cell adhesion molecules and the beta-catenin pathway, NFkappa B and the proteasome, matrix digestion, proteases, and tissue protease inhibitors, and angiogenesis inhibitors [7].

\section{Approaches used to reduce atherosclerosis}

The common approaches such as reduction of oxidative stress by eliminating cigarette smoking, dietary fat intake and administering antioxidant therapeutics, using anti-inflammatory agents to reduce chemokine, cytokine, and growth factor signaling, antiproliferative drugs 
targeting growth factor receptors to reduce cell proliferation (i.e. postangioplasty restenosis in coronary and carotid atherosclerosis). In addition, reduction in excess matrix digestion caused by metalloprotease hyperactivity reduced nuclear factor-kappa B signaling with proteasome inhibitors and use of anti-angiogenesis strategies to delay plaque expansion in atherosclerosis and cancer invasion and metastasis [7].

Some herbs have been reported to show anti-atherosclerotic effects by inhibiting the release of free radicals, hypocholesterolemic effect and through their effect on adhesion molecules leading to inhibition of the inflammatory atherosclerotic process [12]. Oral administration of taurine to rats also showed antioxidant and anti-inflammatory activity by modulating oxidants and resulting in reduced oxidative stress and also showed anti-atherosclerotic activity in hypercholesterolemiainduced atherosclerosis [13].

Recently, in the past decades, stem cells are found to have a significant role in the treatment of a variety of diseases such as hematological disorders, cancer, neurological, cardiac, and nephrological disorders. The role of adult and embryonic stem cell to treat many disorders including Type 1 diabetes mellitus, Parkinson's disease, Huntington's disease, cardiac failure, muscle damage, and liver disorders [14] has been well understood because stem cells have the ability to differentiate into a variety of cells to replace dead cells or to repair tissue. Stem cells have the unique capability to transform and replenish different tissue types in the presence of mechanical force, cytokines, and physical factors [15]. Numbers of therapies are available for heart failure including medical, surgical, and stem cell or progenitor cell therapy, but cell therapy has become a new potential cardiovascular therapeutic tool in recent years [16]. Some findings suggested that stem cells can replace damaged heart cells and restore cardiac function [17]. Cardiac stem cell therapy was first reported in 2001, [18] in a 46-yearold patient who received autologous bone marrow mononuclear cells by a percutaneous transluminal catheter placed in the infarct-related artery and after 10 weeks of administration, the transmural infarct area had been reduced from $24.6 \%$ to $15.7 \%$, while ejection fraction, cardiac index, and stroke volume had increased by $20-30 \%$.

\section{Process of plaque formation}

The main events in the development of atherosclerotic lesions include:

1. Vascular injury: Injured endothelial cells have impaired vasodilator function.

2. Monocyte recruitment, activation of a spectrum of pro-inflammatory functions: Cytokines and complement factor fragments can increase monocyte adhesion and chemotaxis and therefore provide a means for additional monocyte recruitment to the lesion.

3. Macrophage development and foam cell formation: Macrophages and foam cells secrete products that may accentuate the further development of atherosclerotic lesions. Within the advanced atherosclerotic lesion, macrophages can release reactive oxygen species and matrix-degrading proteinases. These enzymes can remodel the arterial matrix, facilitate cellular migration, and likely contribute to the compensatory enlargement characteristic of the growing plaque. Macrophages can die in lesions, in some cases by apoptosis, releasing oxidized cholesterol phospholipids, among other substances that may promote further endothelial injury.

4. Lipid deposition and platelet activation: Smooth muscle cell (SMC) migration and proliferation, and synthesis and deposition of extracellular matrix and fibrous tissue. The result is a platelet aggregation, in response to exposure of the subendothelium, thrombus formation, and increased lipid accumulation within the intima, all of which may aggravate lesion progression.

\section{ROLE OF STEM CELL IN TREATMENT OF ATHEROSCLEROSIS}

\section{Embryonic stem cells and its therapeutic potential}

Embryonic stem cell has impressive self-renewal and differentiation potentials; it can be used with great promise in cell and gene therapy applications in the treatment of many disorders [19,20]. Several studies have shown, an ability to differentiate embryonic stem cells into vascular endothelial cells, SMCs and cardiomyocytes in vitro, representing not only an understanding of the developmental process but also a potential cell source for cardiovascular tissue repair [21].

Japan has successfully made heart pacemaker cells using the embryonic stem cells of mice and this kind of achievement can lead to breakthroughs in treatment for arrhythmia and can reduce the need for electronic pacemakers in human patients [22]. One step ahead of Japan, Singapore has shown that human embryonic stem cells can be transformed into heart cells using a "decellularized" heart as a scaffold. For this, researchers stripped mouse heart cells, leaving only the organ's scaffold, and replaced them with stem cells and after 14 days, the stem cells developed into two different types of cells found in the heart. Then, scaffold was implanted back into the mouse where it was observed to develop visible blood vessels, which are critical for the transport of nutrients and oxygen to the heart [23]. In Australia, a green fluorescent protein gene from jellyfish was inserted into growing heart cells derived from embryonic stem cells, and gene turned the heart cells green, making them easily distinguished from the other cell types in the stem cell culture [24]. These studies indicate that it may be possible to direct the differentiation of embryonic stem cells or adult bone marrow cells into heart muscle cell in cell culture [25].

\section{Adult stem cells and its therapeutic potential}

Adult stem cells residing in specific tissues may be used to replace damaged cells. Two significant adult stem cell sources from heart and bone marrow have been identified to treat cardiovascular pathologies. Bone marrow stem cells can grow into many different tissue types, including muscle, cartilage, bone, liver and different types of neurons, and brain cells [26]. The recognition that the adult heart possesses is presence of stem cell compartment that can regenerate myocytes and coronary vessels and thus raised a unique possibility to rebuild dead myocardium after infarction, to repopulate the hypertrophic decompensate heart with new better functioning myocytes and vascular structures, and perhaps, to reverse ventricular dilation and wall thinning [17,27].

\section{SMCs and its therapeutic potential}

SMC-like cells within an injured blood vessel in animal models or human atherosclerotic lesion may be derived from a variety of sources including circulating blood, medial stem cells, and transdifferentiation of endothelial cells in response to vascular injury [28,29]. SMCs migrate from the media into the intima, where they contribute to neointimal formation by turning into foam cells and producing extracellular matrix but today, this theory is being challenged by the growing evidence that stem cells and smooth muscle progenitor cells (SMPs) contribute to arteriosclerosis by differentiating into SMCs in the intima [30]. It was long thought to derive entirely from pre-existing SMCs in the arterial wall, but this understanding has been challenged by the claim that circulating bone marrow-derived SMPs. These are important source of plaque SMCs in human and experimental atherosclerosis, and today this theory is accepted by many cardiovascular researchers and authors [31]. The progenitor stem cells that remain in a state of growth arrest in the body while retaining the capacity to enter the cell cycle on demand and differentiate into progeny [32].

\section{SPCs and its therapeutic potential}

Stem and progenitor cells are immature cells with high self-renewal potential and ability to differentiate into various cell types, bear potential for organ regeneration in various pathological states $[33,34]$ and we can divide stem and progenitor cells in three categories of methodological assessments: (1) Proliferation, self-renewal, and differentiation in culture, (2) expression of various molecular markers, and (3) ability to execute specialized cell effects, for example, to restore the function of damaged tissue/organ after transplantation [35]. It has been demonstrated that hematopoietic stem cells (HSPCs) are a potential source of Smooth muscle cells (SPCs) or endothelial (endothelial progenitor cells [EPCs]) progenitor cells that can differentiate into SMCs or endothelial cells in animal and also be contributing to pathological arterial remodeling in models of postangioplasty restenosis, transplant-associated atherosclerosis, and hyperlipidemia-induced atherosclerosis [36]. 
It was found that injection of well-characterized SPCs significantly limits the development of atherosclerosis in mice, whereas EPCs injection had no effect, but it has been shown SPCs injections promote substantial changes in plaque composition toward a more stable phenotype, both during the development and the progression of atherosclerosis [37]. Neovascularization of plaque-induced by pro-angiogenic stem cells contributes to plaque growth and instability in some cases [38]. In case of both stable and unstable atherosclerosis plaques showed the presence of stem and progenitor cells [39]. Therefore, stem and progenitor cells can not only play a role in tissue revascularization but also participate in atherosclerosis progression, plaque destabilization, and vessel remodeling [40].

\section{EPCs and its therapeutic potential}

According to new findings, the risk of atherosclerosis implicates a lack of EPC-dependent arterial repair in the development of the disease that is secondary to exhaustion of repair-competent EPCs. Some evidence derived from genetic techniques indicates that atherosclerotic lesions may begin to form as arterial repair fails, rather than merely following an arterial injury. Thus, the chronic arterial injury may overwhelm the ability of EPCs to maintain arterial homeostasis, particularly when EPCs capable of arterial repair become exhausted. According to new submission, genes identified using genetic linkage studies and genomewide association studies that are associated with susceptibility to atherosclerosis and related thromboembolic disorders; these genes may be implicated in the control of arterial wall inflammation and EPCmediated tissue repair [41]. However, the level of circulating EPCs and clinically relevant conditions from endothelial dysfunction to atheroma destabilization and ensuing myocardial infarction showed an inverse relationship to exist between them [42].

\section{Mesenchymal stem cells (MSCs) and its therapeutic potential}

The MSCs are derived from bone marrow, adipose tissue and these are multipotent, less-immunogenic cells, isolation, and in vitro maintenance of MSCs is very easy [26]. MSCs relies on their capacity to engraft and survive long-term in the distinctive target tissue and by using animal models, it has been proved that after the syngeneic and/or xenogeneic transplantation of MSCs, donor cells engraft into the various mesenchymal tissue of the recipient animal [43]. Chen demonstrated significant and sustained improvement in global left ventricular ejection fraction, even larger than that detected after infusion of hematopoietic cells $[44,45]$ and this suggesting that MSC infusion triggers in the human heart the formation of new cardiomyocytes and neoangiogenesis [46]. Addition to the above findings, it provided evidence that the intracoronary infusion of MSCs does not produce any cell size related adverse effect, as reported previously [47]. Mesenchyme stem cells are known to migrate to injured tissue and hypoxic tissue through the expression of receptors such as CD44 [48] and CXCR-4 [49].

Diseases including premature aging diseases, diabetes, atherosclerosis, hematopoietic, cardiovascular, musculoskeletal, gastrointestinal, pulmonary, urogenital, ocular, neurodegenerative, and skin disorders are now to cure using therapeutic approaches [50]. MSC transplantation improved end othelial function and plaque formation in high-fat-diet-fed apoE $\mathrm{E}^{-/-}$mice and activation of the Akt/eNOS pathway in endothelium by interleukin-8 (IL-8)/macrophage inflammatory protein -2 is involved in the protective effect of MSCs and this study support to use and clarify the mechanism of MSCs for ameliorating atherosclerosis [51].

Induced pluripotent stem (iPS) cells and its therapeutic potential It was displayed that pluripotent stem cell has characteristics which are artificially derived from adult non-pluripotent cells, by reprogramming their gene expression patterns ex vivo. Shinya Yamanaka's et al. retrovirally introduced four transcription factors: Oct 3/4, Sox2, c-Myc, and Klf4 in both mouse and human fibroblasts to reprogram the somatic cells. This technology covers unparalleled opportunities in regenerative medicine as iPS cells, can differentiate into specific progenitor cell types. This technology offers a great alternative for the source of differentiated human cells for cell therapeutics in regenerative medicine [52]. In past few years, gene therapeutic trials have not been clinically successful against various genetic disorders, due to the paucity and poor quality of adult stem cells in the bone marrow of patients.

\section{HSPCs and its therapeutic potential}

HSPCs are multipotent stem cells bearing potential for cardiovascular regeneration and HSPCs. It is isolated from bone marrow, peripheral blood, and from umbilical cord blood as CD34+ and CD133+ cells, forming several types of well-characterized colonies in culture (erythroid, myeloid, granulocyte-macrophage, etc.) [53]. Correlation among HSPCs, cholesterol synthesis, and atherosclerosis have been proved by research. It has been seen in mice that high cholesterol levels or inactivation of cholesterol efflux transporters have multiple effects on HSPCs and it includes promoting their mobilization into the bloodstream, increasing proliferation, and differentiating HSPCs to the inflammatory monocytes and neutrophils that participate in atherosclerosis. Increased levels of IL-23 stimulate IL-17 production, resulting in granulocyte colony-stimulating factor secretion, which subsequently leads to HSPC release into the bloodstream. Collectively, we can say these findings clearly link elevated cholesterol levels to increased circulating HSPC levels and differentiation to inflammatory cells that participate in atherosclerosis [57].

\section{VSMC and its therapeutic potential}

VSMC can undergo a proliferative response that underlies the development of instant restenosis, bypass graft occlusion, and transplant vasculopathy. In case of atherosclerosis, the vascular SMCs (VSMCs) contribute to vessel wall inflammation and lipoprotein retention, as well as to the formation of the fibrous cap that provides stability to the plaque [55]. New studies emphasized the involvement of inflammation in mediating all stages of atherosclerosis [56]. The process of atherosclerosis involves the proliferation of VSMCs [57].

\section{CONCLUSION}

This review provides a better understanding of the functions of stem cells and yield information to readers to fully understand the development and as well as treatment of atherosclerosis. Factors and molecular mechanisms that might control stem cell differentiation in the phenotypic state of the stem cells associated with vascular injury is clearly explained. The contribution of stem cells to atherosclerosis progression and vascular remodeling after injury in humans is established; however, many details are still unknown. In this review, the contribution of ECSs in cardiovascular tissue repair and role of adult stem cells is explained in the treatment of cardiac failure. Embryonic stem cell, adult stem cell, SPCs, EPCs, MSCs, and VSMCs can be used to treat and reduce atheroma formation. It is assumed that future studies would explore the ambivalent role of stem cells in tissue regeneration and atherosclerosis progression. Therefore, the next step should be an accumulated attempt to understand the biology of the stem cells, which may provide novel perspicacity into the atherogenesis process and at the same time provide insights into for new therapeutic strategies in the prevention and treatment of atherosclerosis.

\section{AUTHOR'S CONTRIBUTIONS}

The two authors contributed equally to this work.

\section{CONFLICTS OF INTEREST}

The authors do not have any conflicts of interest to declare.

\section{REFERENCES}

1. Moore KJ, Tabas I. Macrophages in the pathogenesis of atherosclerosis. Cell 2011;145:341-55

2. Fuster V, Badimon L, Badimon JJ, Chesebro JH. The pathogenesis of coronary artery disease and the acute coronary syndromes (1). N Engl J Med 1992;326:242-50

3. Virmani R, Kolodgie FD, Burke AP, Farb A, Schwartz SM. Lessons from sudden coronary death: A comprehensive morphological classification scheme for atherosclerotic lesions. Arterioscler Thromb 
Vasc Biol 2000;20:1262-75.

4. Spagnoli LG, Mauriello A, Sangiorgi G, Fratoni S, Bonanno E, Schwartz RS, et al. Extracranial thrombotically active carotid plaque as a risk factor for ischemic stroke. JAMA 2004;292:1845-52.

5. Tyndall A, Walker UA, Cope A, Dazzi F, De Bari C, Fibbe W, et al. Immunomodulatory properties of mesenchymal stem cells: A review based on an interdisciplinary meeting held at the Kennedy institute of rheumatology division, London, UK, 31 October 2005. Arthritis Res Ther 2007;9:301.

6. Indian Heart Association Why South Asians Facts Web. Available from: http://www.who.int/cardiovascular_diseases/about_cvd/en/. [Last accessed on 2015 Apr 29].

7. Ross JS, Stagliano NE, Donovan MJ, Breitbart RE, Ginsburg GS. Atherosclerosis: A cancer of the blood vessels? Am J Clin Pathol 2001;116 Suppl: S97-107.

8. Biros E, Karan M, Golledge J. Genetic variation and atherosclerosis. Curr Genomics 2008;9:29-42.

9. Kovacic S, Bakran M. Genetic susceptibility to atherosclerosis. Stroke Res Treat 2012;2012:362941.

10. Williams KJ, Feig JE, Fisher EA. Cellular and molecular mechanisms for rapid regression of atherosclerosis: From bench top to potentially achievable clinical goal. Curr Opin Lipidol 2007;18:443-50.

11. Davies MJ, Richardson PD, Wool FN, Katz DR, Mann J. Risk of thrombosis in human atherosclerotic plaques: Role of extracellular lipid, macrophage, and smooth muscle cell content. Br Heart $\mathrm{J}$ 1993;69:377-81.

12. Elmorsy EA, Elesawy BH, Baz HA. Effect of black cumin (Nigella sativa) powder on serum lipid profile, Malondialdhydes, Nitrites, SICAM-1 and SVCAM-1 in experimentally induced atherosclerosis. Int J Pharm Pharm Sci 2015;7:412-7.

13. Harsha S, Ankita J, Harsha L, Deepak B. Anti-oxidative, antiinflammatory and anti-atherosclerotic effect of taurine on hypercholesterolemia induced atherosclerotic rats. Int J Pharm Pharm Sci 2018;10:145-50.

14. Tam CS, Khouri I. The role of stem cell transplantation in the management of chronic lymphocytic leukaemia. Hematol Oncol 2009;27:53-60.

15. Mathur A, Martin JF. Stem cells and repair of the heart. Lancet 2004;364:183-92.

16. Dimmeler S, Zeiher AM, Schneider MD. Unchain my heart: The scientific foundations of cardiac repair. J Clin Invest 2005;115:572-83.

17. Beltrami AP, Urbanek K, Kajstura J, Yan SM, Finato N, Bussani R, et al. Evidence that human cardiac myocytes divide after myocardial infarction. N Engl J Med 2001;344:1750-7.

18. Strauer BE, Brehm M, Zeus T, Gattermann N, Hernandez A, Sorg RV, et al. Intracoronary, human autologous stem cell transplantation for myocardial regeneration following myocardial infarction. Dtsch Med Wochenschr 2001;126:932-8.

19. Pazhanisamy S. Stem cell research: The current progress and prospective applications. Drug Lines 2002;4:1-2.

20. Pazhanisamy S, Jyothi V. Genome architecture, instabilities, stem cells and cancer. J Stem Cell Regen Med 2009;4:1.

21. Gepstein L. Derivation and potential applications of human embryonic stem cells. Circ Res 2002;91:866-76.

22. Available from: http://www.tottori-u.ac.jp/dd.aspx?

23. Available from: http://www.ibn.a-star.edu.sg/

24. Available from: http://www.monash.edu/.

25. In Stem Cell Information. Bethesda, MD: National Institutes of Health, U.S. Department of Health and Human Services; 2009. Available from: http://www.stemcells.nih.gov/info/basics/pages/basics6.aspx). [Last cited on 2015 Mar 03].

26. Jiang Y, Jahagirdar BN, Reinhardt RL, Schwartz RE, Keene CD, OrtizGonzalez XR, et al. Pluripotency of mesenchymal stem cells derived from adult marrow. Nature 2002;418:41-9.

27. Leri A, Kajstura J, Anversa P. Cardiac stem cells and mechanisms of myocardial regeneration. Physiol Rev 2005;85:1373-416.

28. Tintut Y, Alfonso Z, Saini T, Radcliff K, Watson K, Boström K, et al. Multilineage potential of cells from the artery wall. Circulation 2003;108:2505-10

29. Ross R, Glomset JA. The pathogenesis of atherosclerosis (second of two parts). N Engl J Med 1976b;295:420-5

30. Han CI, Campbell GR, Campbell JH. Circulating bone marrow cells can contribute to neointimal formation. J Vasc Res 2001;38:113-9.

31. Bentzon JF, Falk E. Circulating smooth muscle progenitor cells in atherosclerosis and plaque rupture: Current perspective and methods of analysis. Vascul Pharmacol 2010;52:11-20.

32. Ingram DA, Mead LE, Tanaka H, Meade V, Fenoglio A, Mortell K, et al. Identification of a novel hierarchy of endothelial progenitor cells using human peripheral and umbilical cord blood. Blood 2004;104:2752-60.

33. Seiler C. The human coronary collateral circulation. Heart 2003;89:1352-7.

34. Dimmeler S, Burchfield J, Zeiher AM. Cell-based therapy of myocardial infarction. Arterioscler Thromb Vasc Biol 2008;28:208-16.

35. van Os R, Kamminga LM, de Haan G. Stem cell assays: Something old, something new, something borrowed. Stem Cells 2004;22:1181-90.

36. Sata M, Saiura A, Kunisato A, Tojo A, Okada S, Tokuhisa T, et al. Hematopoietic stem cells differentiate into vascular cells that participate in the pathogenesis of atherosclerosis. Nat Med 2002;8:403-9.

37. Zoll J, Fontaine V, Gourdy P, Barateau V, Vilar J, Leroyer A, et al. Role of human smooth muscle cell progenitors in atherosclerotic plaque development and composition. Cardiovasc Res 2008;77:471-80.

38. Di Stefano R, Felice F, Balbarini A. Angiogenesis as risk factor for plaque vulnerability. Curr Pharm Des 2009;15:1095-106.

39. Torsney E, Mandal K, Halliday A, Jahangiri M, Xu Q. Characterisation of progenitor cells in human atherosclerotic vessels. Atherosclerosis 2007;191:259-64.

40. Hristov M, Weber C. Ambivalence of progenitor cells in vascular repair and plaque stability. Curr Opin Lipidol 2008;19:491-7.

41. Goldschmidt-Clermont PJ, Seo DM, Wang L, Beecham GW, Liu ZJ, Vazquez-Padron RI, et al. Inflammation, stem cells and atherosclerosis genetics. Curr Opin Mol Ther 2010;12:712-23.

42. Hill JM, Zalos G, Halcox JP, Schenke WH, Waclawiw MA, Quyyumi AA, et al. Circulating endothelial progenitor cells, vascular function, and cardiovascular risk. N Engl J Med 2003;348:593-600.

43. Allers C, Sierralta WD, Neubauer S, Rivera F, Minguell JJ, Conget PA, et al. Dynamic of distribution of human bone marrow-derived mesenchymal stem cells after transplantation into adult unconditioned mice. Transplantation 2004;78:503-8

44. Chan RJ, Yoder MC. The multiple facets of hematopoietic stem cells. Curr Neurovasc Res 2004;1:197-206.

45. Wollert KC, Meyer GP, Lotz J, Ringes-Lichtenberg S, Lippolt P, Breidenbach $\mathrm{C}$, et al. Intracoronary autologous bone-marrow cell transfer after myocardial infarction: The BOOST randomised controlled clinical trial. Lancet 2004;364:141-8.

46. Nagaya N, Fujii $\mathrm{T}$, Iwase $\mathrm{T}$, Ohgushi $\mathrm{H}$, Itoh $\mathrm{T}$, Uematsu $\mathrm{M}$, et al. Intravenous administration of mesenchymal stem cells improves cardiac function in rats with acute myocardial infarction through angiogenesis and myogenesis. Am J Physiol Heart Circ Physiol 2004;287:H2670-6.

47. Vulliet PR, Greeley M, Halloran SM, MacDonald KA, Kittleson MD. Intra-coronary arterial injection of mesenchymal stromal cells and microinfarction in dogs. Lancet 2004;363:783-4.

48. Sackstein R, Merzaban JS, Cain DW, Dagia NM, Spencer JA, Lin CP, et al. Ex vivo glycan engineering of CD44 programs human multipotent mesenchymal stromal cell trafficking to bone. Nat Med 2008;14:181-7.

49. Wang Y, Deng Y, Zhou GQ. SDF-1alpha/CXCR4-mediated migration of systemically transplanted bone marrow stromal cells towards ischemic brain lesion in a rat model. Brain Res 2008;1195:104-12.

50. Mimeault M, Batra SK. Recent insights into the molecular mechanisms involved in aging and the malignant transformation of adult stem/ progenitor cells and their therapeutic implications. Ageing Res Rev 2009;8:94-112.

51. Lin YL, Yet SF, Hsu YT, Wang GJ, Hung SC. Mesenchymal stem cells ameliorate atherosclerotic lesions via restoring endothelial function. Stem Cells Transl Med 2015;4:44-55

52. Takahashi K, Yamanaka S. Induction of pluripotent stem cells from mouse embryonic and adult fibroblast cultures by defined factors. Cell 2006;126:663-76.

53. Gallacher L, Murdoch B, Wu DM, Karanu FN, Keeney M, Bhatia M, et al. Isolation and characterization of human CD34(-)Lin(-) and CD34(+)Lin(-) hematopoietic stem cells using cell surface markers AC133 and CD7. Blood 2000;95:2813-20.

54. Lang JK, Cimato TR. Cholesterol and hematopoietic stem cells: Inflammatory mediators of atherosclerosis. Stem Cells Transl Med 2014;3:549-52.

55. Dzau VJ, Braun-Dullaeus RC, Sedding DG. Vascular proliferation and atherosclerosis: New perspectives and therapeutic strategies. Nat Med 2002;8:1249-56.

56. Libby P, Ridker PM, Maseri A. Inflammation and atherosclerosis. Circulation 2002;105:1135-43.

57. Schwartz SM, Virmani R, Rosenfeld ME. The good smooth muscle cells in atherosclerosis. Curr Atheroscler Rep 2000;2:422-9. 